\title{
POLYMERASE CHAIN REACTION AND FLUORESCENT IN SITU HYBRIDIZATION USED FOR BOVINE EMBRYO SEXING
}

\author{
Assistant Mihai CENARIU*, PhD \\ Professor loan GROZA*, PhD \\ Professor Liviu BOGDAN*, PhD \\ Emoke PALL*, PhD Student \\ Lecturer Simona CIUPE*, PhD
}

\begin{abstract}
The purpose of this paper was to evaluate the results obtained for bovine embryo sexing using the polymerase chain reaction (PCR) and fluorescent in situ hybridization (FISH) and to compare them in order to decide which of the two methods is more accurate and yields better results while necessitating less effort. We took into consideration the pregnancy rate obtained after the transfer of biopsied embryos, the percentage of correctly sexed embryos evaluated at birth (when the predicted sex was compared with the actual sex of the newborn) as well as other characteristics related to the difficulty of the method, expenses and suitability to a minimally equipped laboratory. We concluded that the polymerase chain reaction is the most accurate and suitable method for sexing preimplantation bovine embryos, being in the same time easier to perform than the fluorescence in situ hybridization.
\end{abstract}

Key words: bovine embryo, biopsy, polymerase chain reaction, fluorescent in situ hybridization

\section{Introduction}

Preselection of the sex of offspring of agriculturally important species has long been an objective of animal breeders (Bredbacka, 2001, Groza et al., 2006, Cenariu et al., 2008). However, not until the advent of technologies such as artificial insemination and embryo transfer has such an approach been considered commercially feasible (Cenariu et al. 2008, Groza et al., 2005). Preliminary studies suggest that methods for sexing embryos do show potential for commercial use (Groza et al., 2006, Lee et al., 2004, Taketo et al., 2005). Embryo sexing will be used in conjunction with embryo transfer and most of the research has been geared to sexing bovine and equine embryos, which can be transferred non-surgically up to the blastocyst stage (Jin and Lloyd, 1997). Sexing methods are classified as either invasive or noninvasive, depending on whether or not a biopsy of embryonic tissue is required (Shea, 1999, Cenariu et al., 2008). Criteria that must be considered in embryo sexing techniques are

\footnotetext{
* University of Agricultural Sciences and Veterinary Medicine, Faculty of Veterinary Medicine, Department of Veterinary Reproduction, Obstetrics and Gynecology, 3-5 Manastur Street, 400372 Cluj-Napoca, e-mail: mcenariu@yahoo.es
}

Cluj Veterinary Journal, 15(1)/2009, pp. 19-23 
the percentage of embryos that can be accurately sexed and the effect that the sexing procedure may have on embryo viability (Taketo et al., 2005, Cenariu et al., 2008).

The purpose of this paper was to evaluate the results obtained for bovine embryo sexing using the polymerase chain reaction (PCR) and fluorescent in situ hybridization (FISH) and to compare them in order to decide which of the two methods is more accurate and yields better results while necessitating less effort.

\section{Material and Methods}

A total number of 152 bovine embryos have been used for sexing, and were divided into 2 batches:

- batch 1, made up of 76 bovine embryos that were sexed using the polymerase chain reaction (PCR);

- batch 2, made up of 76 bovine embryos that were sexed using the fluorescent in situ hybridization (FISH).

The embryos have been non-surgically collected from a batch of 16 Simmental donor cows, following superovulation and artificial insemination. The embryos have been morphologically evaluated using a stereomicroscope and biopsy has been performed in order to collect a small number of blastomeres from the inner cell mass (fig.1). After biopsy, the embryos have immediately been transferred to recipient cows, whose estrous cycle had previously been synchronized with the donor cows.

The blastomeres obtained from batch 1 were submitted to DNA isolation and identification of certain nucleotide sequences found only on the $\mathrm{Y}$ chromosome, using specific primers. In short, the technique consisted of the following:

a. DNA extraction from the blastomeres using proteinase $\mathrm{K}$

b. Biosynthesis of the specific primers:

- One set of primers used for PCR were obtained using

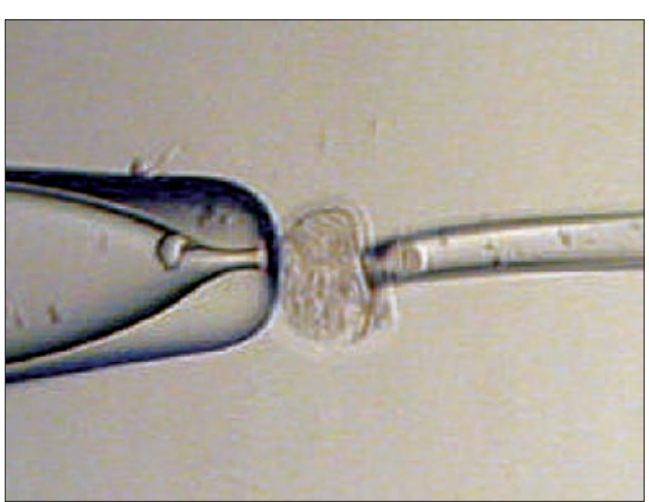

Fig. 1 Aspiration of the blastomeres during embryo biopsy a bovine specific DNA sequence (1715 bovine satellite DNA) in order to show the presence of DNA in all the samples. Thus, the sequence of the two primers was: upstream 5' - TGG AAG CAA AGA ACC CCG CT - 3’ downstream: 5' - TCG TGA GAA ACC GCA CAC TG - 3'

- The second set of primers was obtained using the BRY4a repetitive sequence from the bovine genome that is highly specific for the $\mathrm{Y}$ chromosome and is present only in males. The sequence of the primers was upstream: 5' - CTC AGC AAA GCA CAC CAG AC - 3' and downstream: 5' - GAA CTT TCA AGC AGC TGA GGC - 3'

c. Setting up of the PCR mixture that consisted of $10 \mathrm{ng}$ DNA, 40 pmol of each primer and $45 \mu \mathrm{l}$ of Platinum High Fidelity PCR Supermix (Invitrogen).

d. Amplification of the DNA sequences, using a thermocycler and the following amplification scheme: sample heating at $96^{\circ} \mathrm{C}$ for $3 \mathrm{~min}$., 33 cycles of denaturation at $95^{\circ} \mathrm{C}$ for $1 \mathrm{~min}$., primer annealing at $58^{\circ} \mathrm{C}$ for $1 \mathrm{~min}$. and primer extension at $72^{\circ} \mathrm{C}$ for $1 \mathrm{~min}$. and the final extension at $72^{\circ} \mathrm{C}$ for 5 min.;

e. Electrophoresis of the amplified samples in a 1.5\% agarose gel stained with Gelstar Nucleic Acid gel stain; 
f. Gel examination using a UV transilluminator:

- The presence of a single band corresponding to the bovine specific primers suggested the absence of Y-specific DNA sequences and thus made us classify the embryo as female;

- The presence of two bands one for the bovine specific primers and another for the Y-chromosome specific primers confirmed the presence of a Y-specific DNA sequence and thus made us classify the embryo as male.

The blastomeres obtained from batch 2 were treated with $1 \mu \mathrm{g} / \mathrm{ml}$ vinblastine sulphate for 6 hours in order to induce the chromosomal metaphases, fixed on a slide using methanol and acetic acid and then kept in the freezer until use. The DNA probe was synthesized using the Y-chromosome specific BtY2 gene from which the following primers have been obtained: upstream 5' - TGT TGT GAA GAA GGT GCC CA - 3’ and downstream 5’ - AGT TTG AGG GTG GTT GGT CG - 3’

The primers were used to amplify the male specific DNA sequence obtained after collecting blood from a bull and isolating the DNA from it. The amplification mixture consisted of $5 \mathrm{ng}$ DNA, $1.5 \mathrm{mM}$ of each primer and $45 \mu \mathrm{l}$ Platinum High Fidelity PCR Supermix (Invitrogen). The amplification conditions were: heating the mixture at $95^{\circ} \mathrm{C}$ for 3 minutes followed by 33 cycles of denaturation at $95^{\circ} \mathrm{C}$ for 30 seconds, primer annealing at $55^{\circ} \mathrm{C}$ for 30 seconds and primer extension at $72^{\circ} \mathrm{C}$ for 30 seconds. The final extension consisted of keeping the samples at $72^{\circ} \mathrm{C}$ for 7 minutes. After the amplification, the samples were run on a 1\% agarose gel stained with Gelstar Nucleic Acid gel stain. Following electrophoresis, the gels were examined using a UV trans-illuminator, the bands were cut and the DNA was extracted from the gel in order to obtain the DNA probes used for fluorescent in situ hybridization. The DNA probes were biotinylated by nick translation.

The fluorescence in situ hybridization consisted of the following steps: rehydration of the blastomeres using decreasing concentrations of ethanol, target retrieval using heat and sodium citrate buffer, blastomere digestion using Triton $\mathrm{X}$ and proteinase $\mathrm{K}$, fixation of the blastomeres using paraformaldehyde, application of the in situ frames, application of the hybridization buffer containing 1\% DNA probe, hybridization reaction using a thermocycler (fig. 4) and the following hybridization scheme: $94^{\circ} \mathrm{C}$ for 6 minute and $37^{\circ} \mathrm{C}$ for 16 hours, washing the slides in 3 baths of PBS. The amplification of the fluorescent signal has been achieved using the tyramide signal amplification kit (Perkin-Elmer), which contains streptavidin-HRP, FITC conjugated tyramide and hydrogen peroxide. The slides were subsequently examined under a fluorescence microscope, in order to observe the green fluorescence in male embryos. The pregnancy rates obtained after the transfer of biopsied and sexed embryos were evaluated by rectal palpation, 60 days after the insemination, while the accuracy of embryo sexing was evaluated at birth, when the predicted sex was compared with the morphological sex of the newborn.

\section{Results and Discussion}

The embryo biopsy was successful for all of the embryos and the blastomeres were collected in good conditions.

After performing the experiences in the blastomeres belonging to batch 1, the following results have been obtained:

From the 76 embryos obtained, 70 (92.1\%) had reached the morula stage, while 6 (7.9\%) were in the early blastocyst stage. In what the quality of embryos was concerned, 73 embryos (96\%) were transferable and were used for embryo biopsy while 3 (4\%) could not be used for this purpose. 
After the amplification and gel electrophoresis of the 73 samples, the following results have been obtained:

- 34 samples presented a single 216 bp DNA band when the bovine specific primers were used and no band when the Y-chromosome specific primers were used and thus they were considered to come from female embryos,

- 39 samples presented a 216 bp DNA band when bovine specific primers were used and a 301 bp DNA band when Y-chromosome specific primers were used, thus being considered to come from male embryos.

When evaluating the pregnancy rate in recipient cows in which sexed embryos had been transferred, the following results have been obtained: 28 of 73 females have been diagnosed as pregnant, which represents a percentage of $38 \%$ (chart 1).

At birth, one of the calves obtained presented a different sex than the predicted one (female instead of male) which leads to an accuracy of $96.4 \%$ of the polymerase chain reaction sexing method.

After performing the experiences in the blastomeres belonging to batch 2, the following results have been obtained:

From the 76 embryos obtained, 72 (94.7\%) had reached the morula stage, while $5(5.3 \%)$ were in the early blastocyst stage. In what the quality of embryos was concerned, 74 embryos (97.4\%) were transferable and were used for embryo biopsy while $2(2.6 \%)$ could not be used for this purpose.

The fluorescent in situ hybridization and the amplification of the fluorescent signal using the tyramide kit yielded the following results:

- 41 samples presented the characteristic fluorescence and thus we considered the blastomeres to come from male embryos

- 33 samples did not present any fluorescence and thus we considered the blastomeres to come from female embryos.

When evaluating the pregnancy rate in recipient cows in which sexed embryos had been transferred, the following results have been obtained: 30 of 74 females have been diagnosed pregnant, which represents a percentage of $40.54 \%$ (chart 3).

At birth, 4 of the calves obtained presented a different sex from the predicted one (females instead of males) which leads to an accuracy of $86.66 \%$ for the sexing of bovine embryos using the fluorescence in situ hybridization (chart 4).

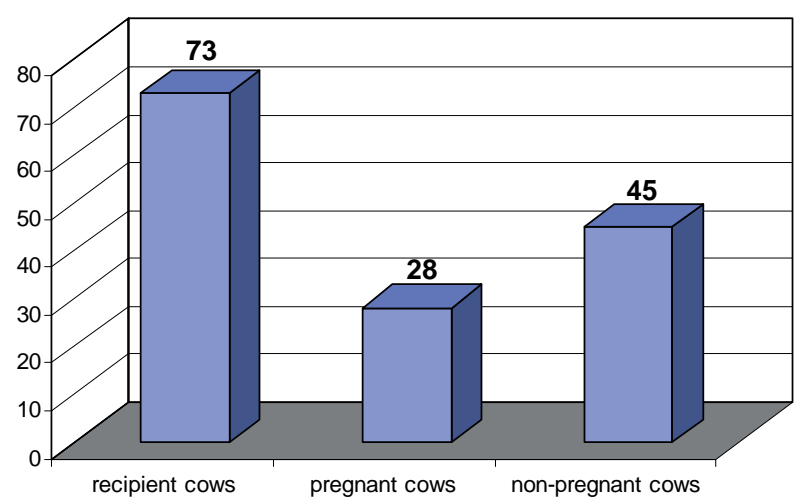

Chart 1. Pregnancy rate in batch 1

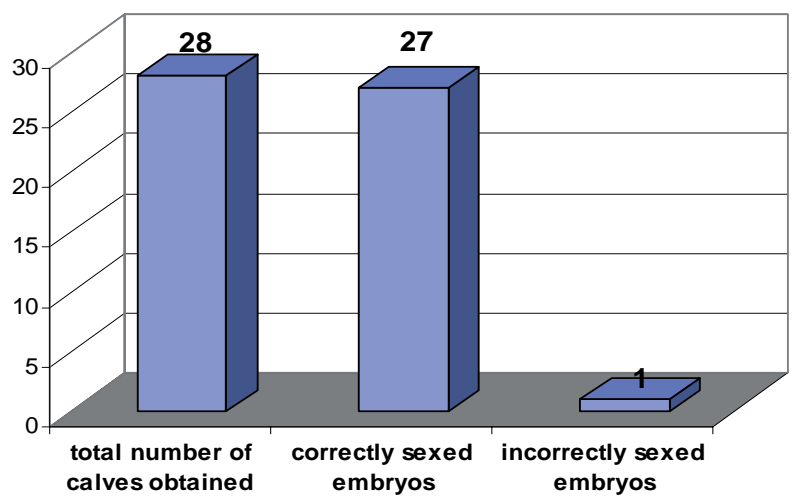

Chart 2. Accuracy of the PCR method of bovine embryo sexing

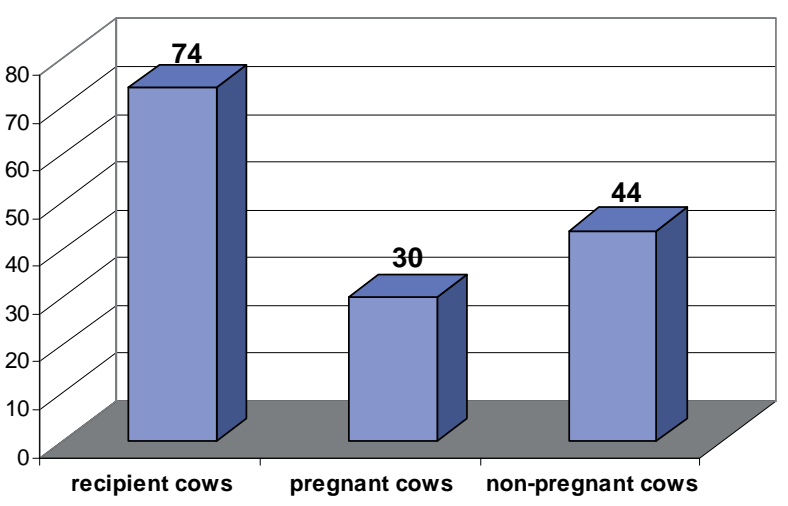

Chart 3. Pregnancy rate obtained in batch 2 
Comparing the results obtained for the two methods of bovine embryo sexing, several observations can be made. First of all, the pregnancy rates obtained after transferring the biopsied embryos is almost similar for the two batches (around 40\%), which is explainable as the biopsy technique did not differ between the two sexing methods. Improvements of the biopsy technique have to be made in the future in order to reduce the damage of the embryos and to be able to obtain higher pregnancy rates. In what the accuracy of the

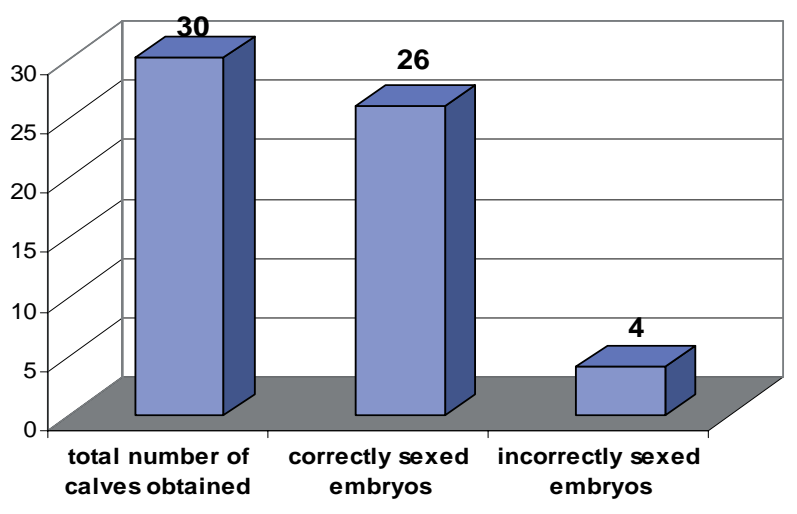

Chart 4. Accuracy of the FISH bovine embryo sexing method two methods is concerned, the PCR sexing yielded better results than the FISH method, being at the same time easier to perform and requesting lower expenses for materials and equipments.

\section{Conclusions}

1. The pregnancy rates obtained after transferring the biopsied embryos were of $38 \%$ in batch 1 and $40.54 \%$ in batch 2 , being almost similar as the biopsy technique did not differ between the two sexing methods.

2. The accuracy of the PCR method of bovine embryo sexing was of $96.4 \%$, one of the embryos having a different sex than the predicted one.

3. The accuracy of the FISH method of bovine embryo sexing was of $86.66 \%$, four of the embryos having a different sex than the predicted one.

4. The PCR sexing of bovine embryos yielded better results than the FISH method, being at the same time easier to perform and requesting lower expenses for materials and equipments.

5. Improvements of the biopsy technique have to be made in the future in order to reduce the damage of the embryos and to be able to obtain higher pregnancy rates.

6. We recommend the commercial use of PCR sexing kits for bovine embryo sexing, the FISH method being appropriate only for research purposes.

\section{References}

1. Bredbacka P. - Progress on methods of gene detection in preimplantation embryos. Theriogenology $\mathbf{5 5}$ pp 23-34, 2001.

2. Cenariu M., I. Groza, R. Al. Pop, Brînduşa Stegeran, Emoke Pall, Laura Cătană, A. Bartoş - Bovine embryo sexing using the fluorescence in situ hybridization (FISH), Bulletin of the University of Agricultural Sciences and Veterinary Medicine Cluj-Napoca, Veterinary Medicine, 65 (2)/2008, ISSN 1843-5270, pp 109-113, 2008.

3. Cenariu M., I. Groza, Simona Ciupe, Brânduşa Stegeran, Emoke Pall, Cătană Laura, A. Bartoş - Bovine embryo sexing using the polymerase chain reaction (PCR), Scientific Papers USAMV Iaşi, Veterinary Medicine, vol. 51/2008, ISSN 1454-7406, pp 252-256, 2008

4. Groza I., L. Bogdan, I. Morar, Simona Ciupe, R. Pop, M. Cenariu, C. Peştean - Cercetari privind variantele de inovulare a embrionilor la bovine, Clujul Medical Veterinar, nr.8, pp 8-12, 2005.

5. Groza I. şi col. - Ginecologie, Andrologie şi Obstetrică Veterinară, Ed. Academiei Române, Bucureşti, 2006

6. Lee J.H., J. H. Park S.H. Lee, C.S. Park, D. I. Jin - Sexing using single blastomere derived from IVF bovine embryos by fluorescence in situ hybridization (FISH), Theriogenology 62, pp 1452-1458, 2004

7. Jin L., R.V. Lloyd - In situ hybridization: methods and applications. J.Clin. Lab. Anal. 11(1), pp 2-9, 1997

8. Shea B.E. - Determining the sex of bovine embryos using polymerase chain reaction results: a six-year retrospective study. Theriogenology 51, pp785-797, 1999.

9. Taketo, T., Lee, C.H., Zhang, J., Li, Y., Lee, C.Y., Lau, Y.F. - Expression of SRY proteins in both normal and sex-reversed XY fetal mouse gonads. Dev. Dyn. 233, pp 612-622, 2005. 